\title{
A novel high frequency signal injection strategy for self-sensing control of electric AC machine drives
}

\author{
$1^{\text {st }}$ Amir MESSALI \\ Centrale Nantes, LS2N UMR \\ CNRS 6004, Nantes, France \\ amir.messali@ec-nantes.fr
}

\author{
$2^{\text {nd }}$ Med Aassad HAMIDA \\ Centrale Nantes, LS2N UMR \\ CNRS 6004, Nantes, France \\ mohamed.hamida@ec-nantes.fr
}

\author{
$3^{\text {rd }}$ Malek GHANES \\ Centrale Nantes, LS2N UMR \\ CNRS 6004, Nantes, France \\ malek.ghanes@ec-nantes.fr
}

\author{
$4^{\text {th }}$ Mohamad KOTEICH \\ Renault Group, Technocentre \\ Guyancourt, France \\ mohamad.koteich@renault.com
}

\begin{abstract}
In this paper, an improved high frequency (HF) injection method coupled with a robust observer is proposed for Interior Permanent Magnet Synchronous Motor (IPMSM), to estimate position, speed and acceleration without mechanical sensors. The contribution is twofold. First, a square-wave HF signal injection method with a new demodulation process is adopted to improve the performances of the self-sensing control. Second, an extended sliding mode observer combined with the HF method is proposed to get over the knowledge of machine parameters (inductances, inertia, frictions,...) and to improve the estimation error in transient modes, which are the drawbacks of existing tracking algorithms (phase-locked loop (PLLs), mechanical system observers, inverse of tangent). Simulation and experimental results are provided to show the effectiveness and the robustness of the proposed strategy in the framework of electric propulsion used in automotive applications.
\end{abstract}

Index Terms-Synchronous machine, HF signal injection, tracking algorithm, sliding mode observer, self-sensing conrol.

\section{INTRODUCTION}

Over last two decades, due to their high performance and efficiency, Interior Permanent Synchronous Machines have known an important interest in many industrial applications. IPMSM control requires mechanical sensors to obtain the rotor position information. However, these elements (position sensors, rotational transducers) are not only expensive, bulky and sensitive to the environment, but they reduce the system reliability. These drawbacks motivate researchers to immigrate to the self-sensing control of electrical machines. In the literature, several techniques are proposed for rotor position and speed estimation of synchronous machine. These methods are classified into two main categories: category 1, model based techniques: Artificial intelligence techniques [1], nonlinear observers [2], MRAS [3] methods. category 2, saliency based techniques by injected a HF signal [4], [5]. In this paper, category 2 is considered. Compared to category 1, this technique offers attractive advantages such as the robustness of the position and speed estimation when the machine is running at low speed (including zero velocity). However, it is still dependent on the knowledge of certain parameters like machine inductances, the magnitude and the frequency of the injected HF signal. Moreover, this technique is often

This work was supported by the project Chair between Renault and Centrale Nantes about performances of electric vehicles propulsion. validated over a reduced operating ranges of the machine, namely the low speed operation. Furthermore, the machine magnetic saturation presence (due to high speed, high torque) affects strongly the rotor position/speed estimation process, machine saturation models [6] proposed in the literature are not sufficiently adequate. Several techniques based on HF signal injection have been developed in the literature. The squarewave signal injection technique presents several advantages compared to other techniques such as filters removal [7]. In this work, square-wave technique is used with a measurement of the current shifted by $\frac{\pi}{4}$ with respect to the injection frame. This technique is enhanced by a new demodulation process (reducing harmonics and simplicity of implementation) to improve the rotor position and speed estimation. Moreovor, a robust position, speed and acceleration estimation strategy for IPMSM without mechanical sensors is proposed to avoid the knowledge of machine parameters (inductances, inertia, frictions,...) and cover an extended operating ranges of the machine (medium and high speeds operating modes with full torque). The rest of this paper is organized as follows. The IPMSM modeling with HF signal injection is presented in section II. Section III introduces the square-wave injection method and the new demodulation process. The proposed estimation procedure is detailed in Section IV. In Section V, the performance and the robustness of the proposed estimation strategy are highlighted by simulation and experimental results with an electrical propulsion profile used in automotive applications. In this section, a comparative analysis for the proposed strategy with the classical PLL is shown. Conclusions and perspectives are given in section VI.

\section{MATHEMATiCAL MOdELS}

In this section, the HF voltage and flux-current IPMSM models [8] are presented in stator and rotor reference frames.

\section{A. Notations}

The complex notation $\underline{Z}_{b}^{a}$ is adopted in this paper where the subscript $b$ refers to a stator quantity, whereas the superscript $a$ is either a rotor $(r)$ or stator $(s)$ quantity that allows to know whether the quantity is expressed in the stator $(\alpha \beta)$ or in the rotor $(d q)$ reference frame. 


\section{B. High Frequency IPMSM models}

HF models are obtained by considering following assumptions:

- The HF stator impedance $\left(Z_{s}=R_{s}+j \omega_{c} L_{s}\right)$ of the machine is dominated by self-stator reactance $\left(R_{s}<j \omega_{c} L_{s}\right)$ , it means that the influence of the stator resistance is neglected.

- In the rotor reference frame, the two axes $(d q)$ being magnetically decoupled from each others.

- The rotating Back-EMF is neglected.

$\mathrm{HF}$ voltage and flux-current models are given by [9]:

1) Stationary frame voltage model: By using Ohm's Law and assumptions below, the voltage model on stator reference is given as follows

$$
v_{s}{ }^{s} \simeq \frac{\Delta \psi_{s}{ }^{s}}{\Delta T}
$$

where, $\psi_{s}^{s}$ is the HF stator flux and $\Delta T$ is the HF voltage injection period.

2) Stationary frame flux-current model: The HF magnetic flux in the rotating frame is given by:

$$
\psi_{-}^{r}=\psi_{d}+j \psi_{q}
$$

where,

$$
\psi_{d}=L_{d} i_{d}, \quad \psi_{q}=L_{q} i_{q}
$$

with $L_{d}, L_{q}$ are respectively $d-q$ axes inductances.

By replacing (3) in (2), the following expression is obtained

$$
\psi_{s}{ }^{r}=L_{0} \underline{i}_{\underline{s}}{ }^{r}+L_{2} i_{\underline{s}}{ }^{r *}
$$

where,

$$
L_{0}=\frac{L_{d}+L_{q}}{2}
$$

and

$$
L_{2}=\frac{L_{d}-L_{q}}{2}
$$

are respectively the average and the differential inductances. By using (4) and Park transformation, the HF magnetic flux in the stationary frame is deduced

$$
\psi_{\underline{s}}{ }^{s}=L_{0} i_{\underline{s}}{ }^{s}+L_{2} i_{\underline{s}}{ }^{s *} e^{j 2 \theta} .
$$

Variations of inductances $L_{d}$ and $L_{q}$ are supposed negligible against the dynamic variations of $i_{d}$ and $i_{q}$ currents, consequently dynamic of the equation (7) is obtained by using Taylor's theorem first derivative:

$$
\Delta \psi_{s}{ }^{s}=L_{0} \Delta \underline{i}_{s}{ }^{s}+L_{2} \Delta \underline{i}_{s}{ }^{s *} e^{j 2 \theta} .
$$

From (8), the current dynamic expression is deduced

$$
\underline{\Delta}_{s}{ }^{s}=\frac{1}{L_{0}^{2}-L_{2}^{2}}\left(L_{0} \Delta \underline{\psi}_{s}{ }^{s}-L_{2} \Delta \underline{\psi}_{s}{ }^{s *} e^{j 2 \theta}\right) .
$$

As discussed in the introduction, several HF injection methods are developed in the literature, in this paper, the square-wave injection method [10] is adopted with a new demodulation process to introduce the proposed strategy of rotor position, speed and acceleration estimation. Note that these contributions can be generalized to all HF injection methods.

\section{HF SQUARE-WAVE VOLTAGE INJECTION METHOD}

The high frequency square-wave signal injection technique is developed for self-sensing control of AC machines [10]. This technique offers numerous advantages compared to conventional methods (rotating [11], pulsating [12], INFORM [13]...) such as:

- Remove low pass filters to avoid delays on rotor position and speed estimation.

- Facilitate the frequency separation between the injected frequency and the fundamental one due to the too high injected frequency.

- Increase bandwidths of both the tracking observer and the regulator.

- Reduce the stator resistance and the back EMF effects due to the high injected frequency.

However, due to the very high frequency of the injected signal, this technique generates non negligible acoustic noises and requires sensitive current sensors.

\section{A. Injected voltage}

In this part, the HF injected voltage signal is applied in a fixed direction, and added to the $\mathrm{d}$ axis output voltage (see Fig. 2). The square-wave voltage vector in the estimated $(\hat{d} \hat{q})$ frame can be expressed as

$$
v_{s}^{\hat{r}}=\left[\begin{array}{l}
v_{\hat{d}} \\
v_{\hat{q}}
\end{array}\right]=V_{c}(-1)^{k}\left[\begin{array}{l}
1 \\
0
\end{array}\right]
$$

where $V_{c}$ is the magnitude of the injected voltage and $\mathrm{k}$ is the index of PWM cycles. Fig. 1 shows characteristics of the square-wave voltage that is applied in the estimated reference frame.

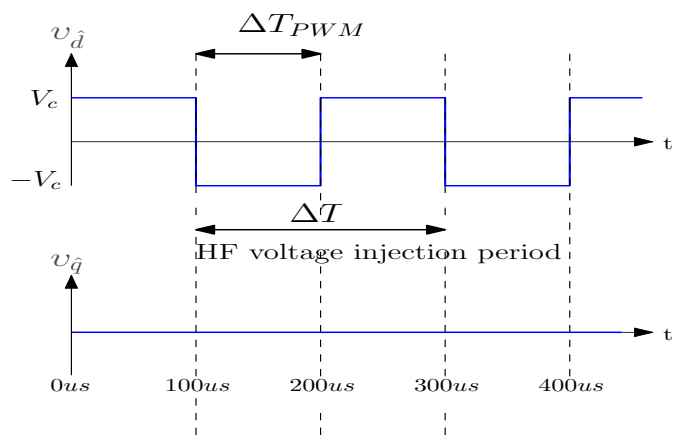

Figure 1. HF voltage injection square-wave vector

From (10), the HF injected voltage in the stator reference frame is expressed

$$
\left[\begin{array}{l}
v_{\alpha} \\
v_{\beta}
\end{array}\right]=V_{c}(-1)^{k}\left[\begin{array}{l}
\cos (\widehat{\theta}) \\
\sin (\widehat{\theta})
\end{array}\right] .
$$

\section{B. Current resulting from the injected voltage}

The stator flux generated by the high frequency signal injection can be obtained by using equations (1) and (11)

$$
\Delta \underline{\psi}_{s}{ }^{s}=V_{c}(-1)^{k} \Delta T\left[\begin{array}{c}
\cos (\widehat{\theta}) \\
\sin (\widehat{\theta})
\end{array}\right]
$$


From (12) and (9), the high frequency stator current is expressed by

$$
\Delta_{-}{ }_{-}^{s}=\frac{V_{c}(-1)^{k} \Delta T}{\left(L_{0}^{2}-L_{2}^{2}\right)}\left(L_{0} e^{j \hat{\theta}}-L_{2} e^{j(2 \theta-\hat{\theta})}\right)
$$

From equation (13), it can be seen that only the HF negative current component $\frac{V_{c}(-1)^{k} \Delta T L_{2}}{\left(L_{0}^{2}-L_{2}^{2}\right)} e^{j(2 \theta-\hat{\theta})}$ contains the saliency information located in the IPMS machine rotor.

\section{Demodulation process and error extraction}

Numerous heterodyning process have been proposed in the literature [8], in this paper, a new demodulation process is proposed, in addition to its control performance and simplicity of the implementation [14], this method improves the rotor position and speed estimation process.

Fig. 2 shows the square-wave vector that is applied in the estimated reference frame and the current measurement frame.

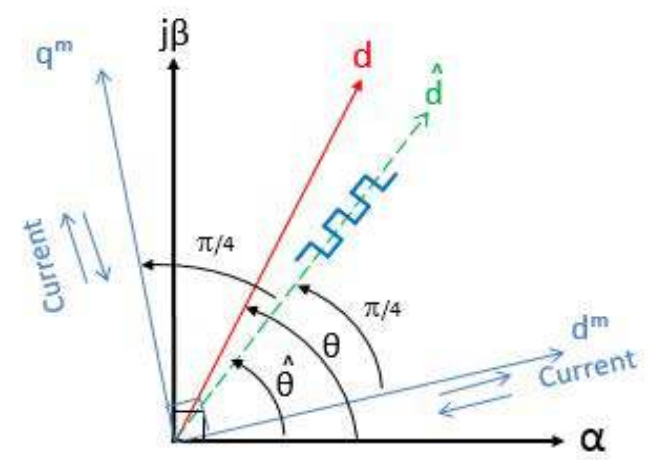

Figure 2. HF square-wave voltage injection technique with a measurement of the current shifted by $\frac{\pi}{4}$ from the estimated frame

The current expression in the reference frame shifted by $\frac{\pi}{4}$ with respect to the injection frame is given as follows

$$
\Delta i_{-}{ }^{m}=I_{c p} e^{j \frac{\pi}{4}}-I_{c n} e^{j\left(2 \theta-2 \hat{\theta}+\frac{\pi}{4}\right)}
$$

where,

$$
I_{c p}=\frac{L_{0} V_{c}(-1)^{k} \Delta T}{\left(L_{0}^{2}-L_{2}^{2}\right)}=\frac{\left(L_{d}+L_{q}\right) V_{c}(-1)^{k} \Delta T}{2 L_{d} L_{q}}
$$

and

$$
I_{c n}=\frac{L_{2} V_{c}(-1)^{k} \Delta T}{\left(L_{0}^{2}-L_{2}^{2}\right)}=\frac{\left(L_{d}-L_{q}\right) V_{c}(-1)^{k} \Delta T}{2 L_{d} L_{q}}
$$

are respectively the magnitude of the high frequency positive component and the high frequency negative component of the stator current. The equation (14) can be written as follows

$$
\Delta i_{s}^{m}=\left[\begin{array}{c}
\Delta i_{d}^{m} \\
\Delta i_{q}^{m}
\end{array}\right]=\left[\begin{array}{l}
I_{c p} \frac{\sqrt{2}}{2}-I_{c n} \cos \left[2(\theta-\hat{\theta})+\frac{\pi}{4}\right] \\
I_{c p} \frac{\sqrt{2}}{2}-I_{c n} \sin \left[2(\theta-\hat{\theta})+\frac{\pi}{4}\right]
\end{array}\right]
$$

(17) can be written

$$
\left[\begin{array}{l}
\Delta i_{d}^{m} \\
\Delta i_{q}^{m}
\end{array}\right]=\left[\begin{array}{l}
I_{c p} \frac{\sqrt{2}}{2}-I_{c n} \frac{\sqrt{2}}{2}[\cos (2(\theta-\hat{\theta}))-\sin (2(\theta-\hat{\theta})) \\
I_{c p} \frac{\sqrt{2}}{2}-I_{c n} \frac{\sqrt{2}}{2}[\cos (2(\theta-\hat{\theta}))+\sin (2(\theta-\hat{\theta}))
\end{array}\right]
$$

The estimation position error expression $(\theta-\hat{\theta})$ can be deduced by computing the current difference quantities $\Delta i_{q}^{m}-\Delta i_{d}^{m}$

$$
\begin{aligned}
\varepsilon & =\Delta i_{q}^{m}-\Delta i_{d}^{m} \\
& =-I_{c n} \sqrt{2} \sin [2(\theta-\hat{\theta})]
\end{aligned}
$$

In addition to advantages of classical demodulation, the proposed method introduced in (19) reduces harmonics on the rotor position estimation error that improves the robustness of the estimation. To ensure the positivity of the injected voltage, the equation (19) multiplied by $\left(v_{\hat{d}}\right)$ becomes

$$
\begin{aligned}
\varepsilon & =v_{\hat{d}}\left(i_{q}^{m}-i_{d}^{m}\right) \\
& =-V_{c}(-1)^{k} I_{c n} \sqrt{2} \sin [2(\theta-\hat{\theta})] \\
& =-I_{c n}^{*} \sin 2(\theta-\hat{\theta})
\end{aligned}
$$

where,

$$
I_{c n}^{*}=\frac{\left(L_{d}-L_{q}\right)\left[V_{c}(-1)^{k}\right]^{2} \Delta T \sqrt{2}}{2 L_{d} L_{q}}
$$

Finally, $\varepsilon$ can be expressed as:

$$
\varepsilon=-I_{c n}^{*} \sin (2(\theta-\hat{\theta})) .
$$

The above developed estimation process based on squarewave voltage injection technique is summarized in Fig. 3. In this figure, the high-pass filter (HPF) is used to remove the fondamental frequency, the block "Robust estimation of $\theta$ and $\omega$ and $\alpha^{\prime \prime}$ which represents the second and the principle contribution of this paper, will be the subject of the next section.

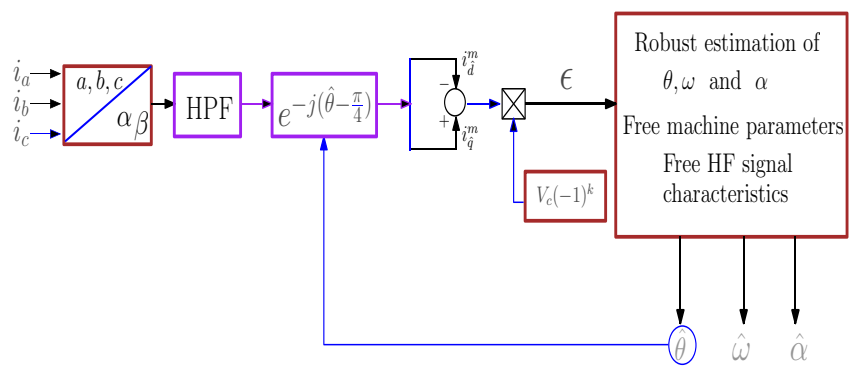

Figure 3. Scheme of rotor position, speed and acceleration estimation based on square-wave injection technique

\section{Proposed Robust Rotor POSITION, SPEED AND ACCELERATION ESTIMATION BASED ON SLIDING MODE OBSERVER}

As mentioned on the introduction, existing tracking algorithms (the inverse of tangent [15], the PLL [16], and the mechanical observer [17] ...) are used to estimate the rotor position/speed based on the error expression given in (22). However, this error expression depends on the knowledge of the current negative sequence $I_{c n}^{*}$ defined in (21). As $I_{c n}^{*}$ depends both on machine parameters (inductances $L_{d}, L_{q}$ in (5)-(6)) and injected signal characteristics (magnitude $V_{c}$ and frequency $\frac{1}{\Delta T}$ in (21)), all these tracking algorithms are sensitive to $I_{c n}^{*}$ variations (due to the magnetic saturation). Moreover, the mechanical observer requires the good knowledge of mechanical parameters (Inertia $\mathrm{J}$, frictions $K_{f} \ldots$ ) and 
the load torque, these parameters vary significantly according to several unknown factors. PLLs are also not adapted to high speed variations (sensitive to the acceleration). The rotor position/speed estimation in all speed/torque ranges independently of machine parameters (inductances, inertia...) and injected signal characteristics (magnitude and frequency) is still an open problem. The proposed strategy describes the rotor position, speed and acceleration estimation for IPMSM which allows to:

- Replace classical tracking algorithms by sliding mode observer.

- Avoid the knowledge of the gain $I_{c n}^{*}$ and have free machine parameters estimation.

- Estimate the acceleration that compensate the speed error in the acceleration phase, i.e. improve the position/speed estimation.

- Robustify the estimation even in the case of magnetic saturation.

Assumption 1: The variation of the position estimation error $\theta-\hat{\theta}$ is supposed to be small, i.e. $\theta \simeq \hat{\theta}$.

To overcome the $I_{c n}^{*}$ dependency, the novelty of the proposed strategy consists to use only the sign of the position error in the correction term of position, speed and acceleration estimation algorithm, instead of using the position error $\varepsilon$ defined in (22):

$$
\begin{aligned}
\sigma & =\operatorname{sign}(\varepsilon)=\operatorname{sign}\left(-I_{c n}^{*}(\theta-\hat{\theta})\right) \\
& =\operatorname{sign}\left(-I_{c n}^{*}\right) \operatorname{sign}(\theta-\hat{\theta}) .
\end{aligned}
$$

In general case, $-I_{c n}^{*}>0$ because $L_{q}>L_{d}$ and $\left[V_{c}(-1)^{k}\right]^{2} \Delta T>$ 0 , consequently (23) becomes

$$
\sigma=\operatorname{sign}(\Delta \theta)
$$

where $\operatorname{sign}(\Delta \theta)$ is the sigmoid function of the form:

$$
\operatorname{sign}(\Delta \theta):\left\{\begin{array}{l}
1 \text { if } \Delta \theta>0 \\
-1 \text { if } \Delta \theta<0 \\
0 \text { if } \Delta \theta=0
\end{array}\right.
$$

and $\Delta \theta=\theta-\hat{\theta}$. It can be argued that equation (24) doesn't depend on machine parameters $\left(L_{d}, L_{q}, J, K_{f}, \ldots\right)$, injected signal characteristics $\left(V_{c}, \frac{1}{\Delta T}\right)$ and the load torque.

Only the sign of the position error is required to estimate the rotor position, speed and acceleration of IPMSM by the proposed (25)-(26)-(27) finite time sliding mode observer (see Fig. 4)

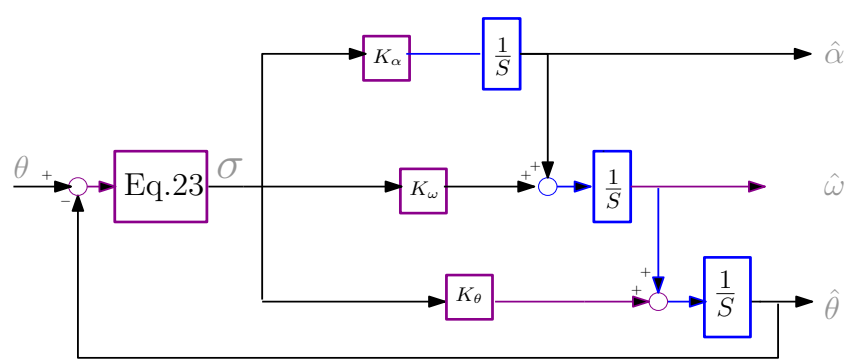

Figure 4. Robust estimation of $\theta, \omega$ and $\alpha$

$$
\begin{aligned}
\dot{\hat{\theta}} & =\hat{\omega}+K_{\theta} \sigma \\
\dot{\hat{\omega}} & =\hat{\alpha}+K_{\omega} \sigma \\
\dot{\hat{\alpha}} & =K_{\alpha} \sigma
\end{aligned}
$$

The mechanical system of the position, the speed and the acceleration used for designing observer (25)-(26)-(27) is given by:

$$
\begin{aligned}
\dot{\theta} & =\omega \\
\dot{\omega} & =\alpha \\
\dot{\alpha} & =0
\end{aligned}
$$

Usually, in the literature, the rotor speed is considered constant. When the dynamic of rotor speed is taken into account, mechanical parameters should be well known. System (28), (29) and (30) overcomes above limitations (constant speed and knowledge of mechanical parameters) by considering the acceleration of the machine.

\section{A. Stability analysis}

For a lack of space, the stability analysis is not given here, it is inspired from [18].

\section{B. Parameters tuning of the proposed strategy}

- The parameters $K_{\alpha}, K_{\omega}$ and $K_{\theta}$ are chosen according to the stability analysis [18]. The injected signal frequency is chosen to be greater than the nominal machine fundamental frequency and less than the switching inverter frequency (to verify the Shannon property, it should be at most half of the switching frequency).

- The injected signal magnitude is chosen small enough in order to avoid torque ripples and the machine warming.

\section{Simulations AND EXPERIMENTAL RESUlts}

To evaluate the performance of the proposed strategy in selfsensing mode, simulations on Matlab/Simulink software and experiments on test bench (see Fig. 6) were carried out. The sampling period is chosen to be $10^{-4} s$ and the PWM frequency is set to $10 \mathrm{kHz}$. The DC voltage is set to $400 \mathrm{~V}$. The motor parameters are given in table I. Injected signal characteristics are chosen: $\omega_{c}=5000 \times 2 \pi(\mathrm{Rad} / \mathrm{s})$ and $V_{c}=3(V)$. Observer parameters values are chosen as follows $k_{\alpha}=200 \mathrm{rads}^{-2}$, $k_{\omega}=110 \mathrm{rad} . \mathrm{s}^{-1}$ and $k_{\theta}=250 \mathrm{rad}$. A representative cycle of drive benchmark shown in the first figure of Fig. 7 is used to validate the performance of the proposed strategy. An arbitrary inductances profiles are introduced (see Fig. 5) to prove that the proposed estimation method is independent from machine parameters. Moreover, an important white noise is added to current measurements. 


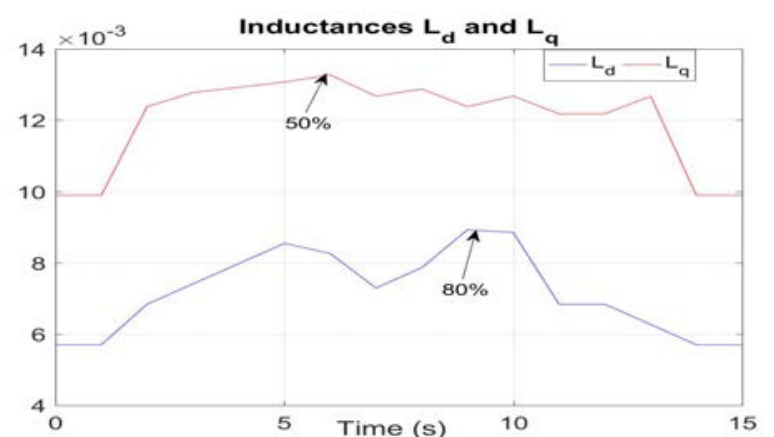

Figure 5. Arbitrary inductance profiles

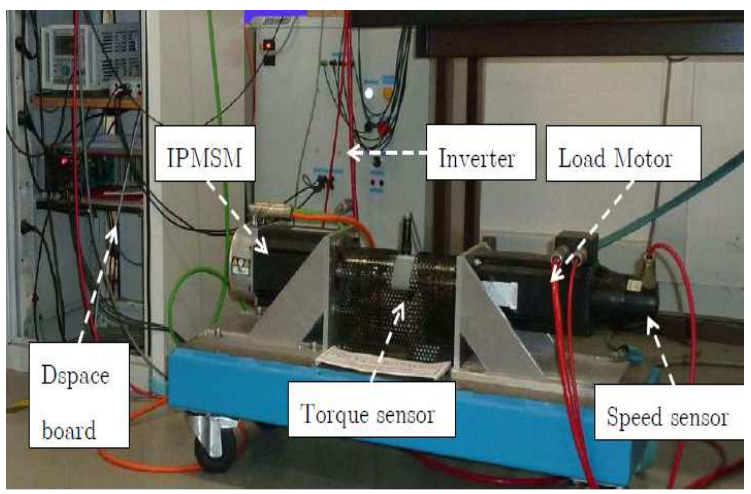

Figure 6. Motor set-up

Simulation (see Fig. 7) and experimental (see Fig. 9) results of the proposed strategy display following variables: the measured and the estimated mechanical speeds, the speed estimation error, the measured and the estimated electrical positions, the electrical position estimation error, the reference and the measured current $i_{d}$, the reference and the measured current $i_{q}$ and the acceleration machine estimation. Note that measurements of position and speed are only given for comparison with estimations of position and speed. The estimated speed tracks very well the measured one even in acceleration phases and the speed error is centered around zero. The acceleration is well estimated which allows to have a good rotor position and speed estimation especially in acceleration phases. The electrical position estimation error is less than \pm 5 degrees. A small noisy position and speed error, that is seen in both simulation and experimental results, is due to harmonics generated by the very high injected frequency, the injected voltage's form (square-wave) and the sliding mode chattering. To reduce the noticed noisy error, the estimation variables can be filtered, however, the use of filters generates delays that affects the estimation process and increase the complexity and the implementation cost. Despite advantages of both square-wave and the proposed strategy, this technique generates non negligible acoustic noises and requires sensitive current sensors.

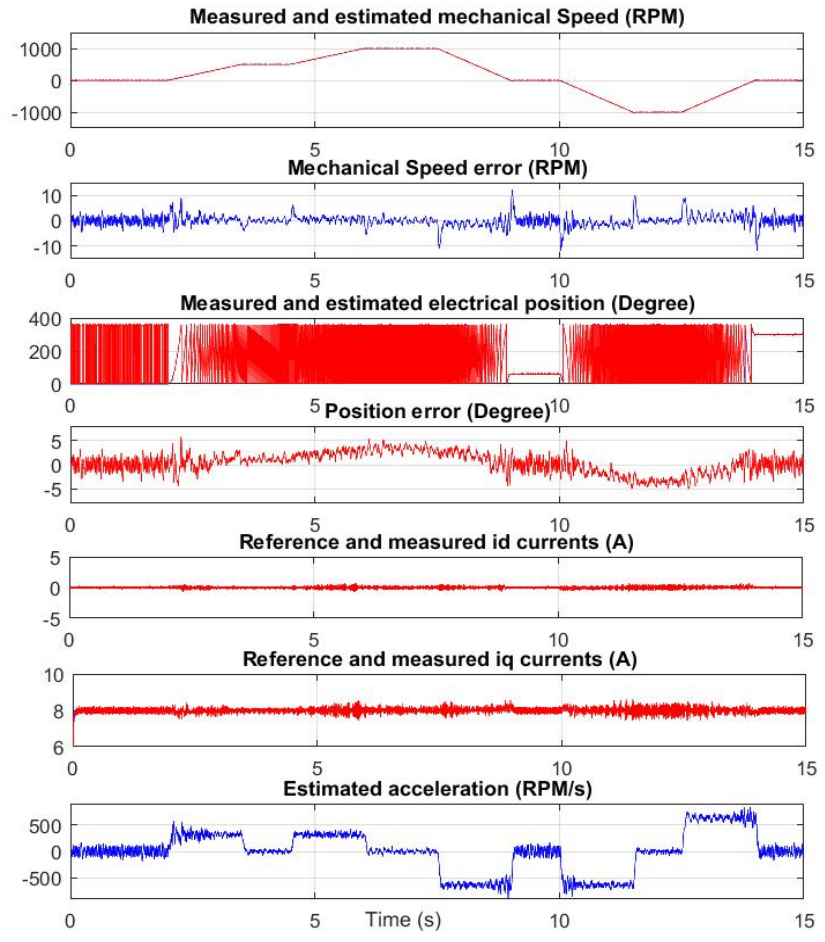

Figure 7. Simulation Results

In the literature, The mostly used tracking observer is the PLL [19], it is evaluated in simulation with and without considering the inductance variations.

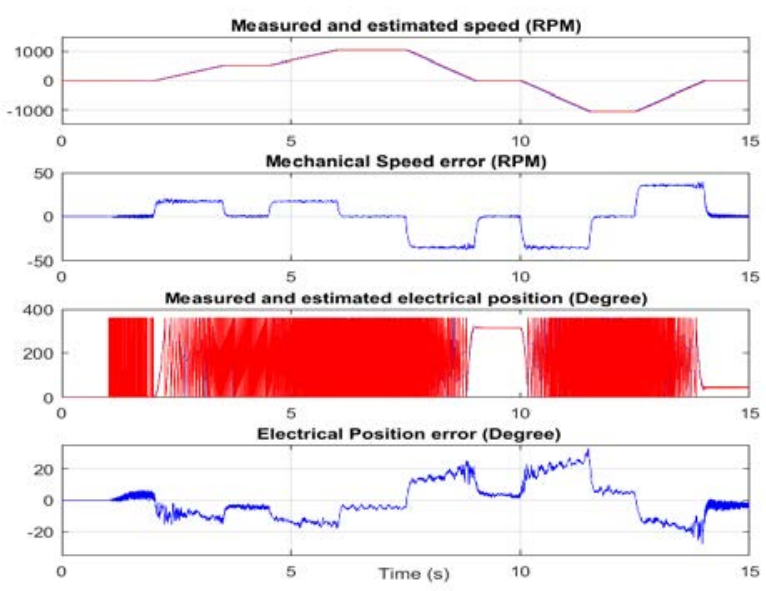

Figure 8. PLL Simulation Results

On the obtained results (see Fig. 8), an important estimation errors can be seen in the rotor position and speed estimation. Once the inductance profiles are considered (see Fig. 5), the PLL algorithm diverges immediately. These results prove that this method is very sensitive to acceleration effects and parameters (inductances) variations. Simulation and experimental results confirm the effectiveness and the robustness of the proposed observer. 


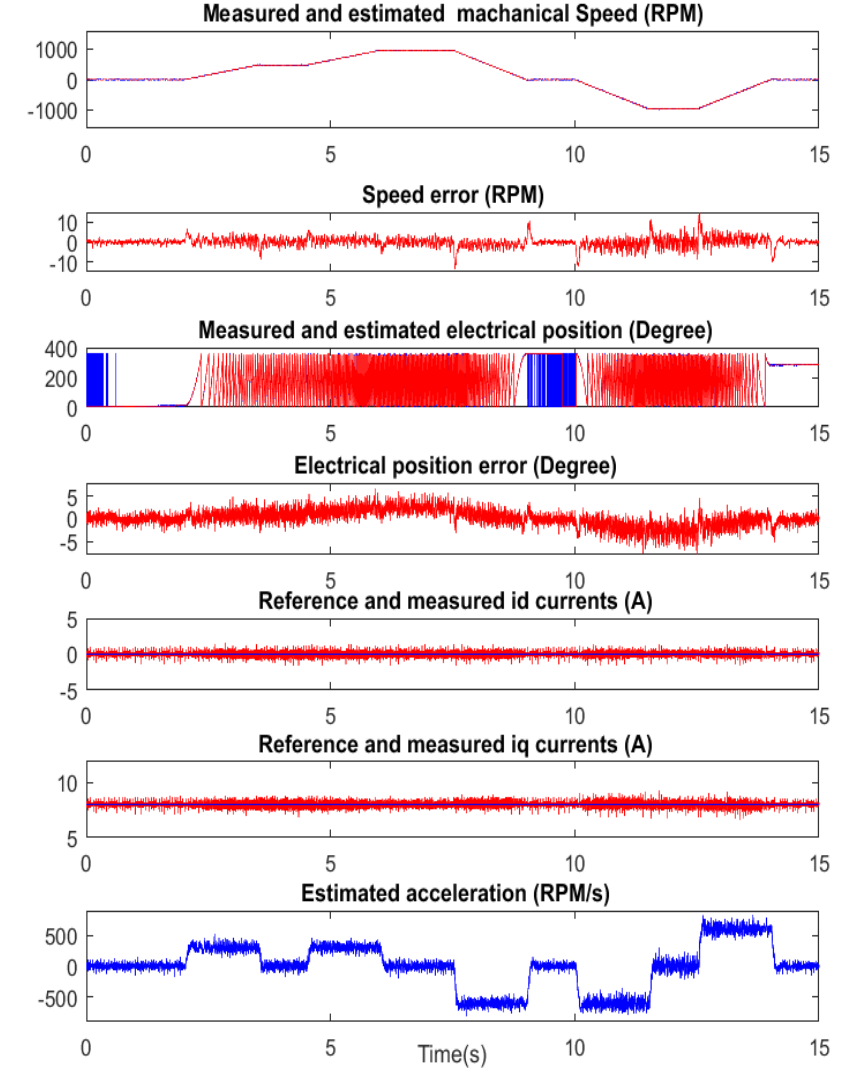

Figure 9. Experimental results

\section{CONCLUSION}

In this paper, a new robust solution for the rotor position, speed and acceleration estimation is developed. A new demodulation process enhanced by the proposed observer are associated to the square-wave signal injection method. Comparative study shown (see Fig. 8) that classical techniques used to extract the rotor position and speed depend on the machine parameters and they are sensitive to acceleration effects. The theoretical development, the simulation and the experimental results prove the no dependency of the proposed strategy on machine parameters and injected signal characteristics. Moreover, estimation results are improved at transient states by considering acceleration estimation. As a consequence, under a self-sensing control, the IPMSM can operate in all speed ranges with full torque. These properties allow the proposed observer to be a strong candidate to replace classical tracking algorithms (PLLs, mechanical observer and inverse of tangent) widely used in the literature. Our next objective is to overcome limitations of the square-wave signal injection method and to deal with the cross saturation problem.

Table I

MOTOR PARAMETERS

\begin{tabular}{|c|c|c|c|}
\hline Speed & $2100 \mathrm{rpm}$ & Torque & $9 \mathrm{Nm}$ \\
\hline$J$ & $0.0073 \mathrm{~kg} . \mathrm{m}^{2}$ & $\Phi_{f}$ & $0.33 \mathrm{~Wb}$ \\
\hline$R_{s}$ & $1.4 \Omega$ & $L_{d}$ & $0.0057 \mathrm{H}$ \\
\hline$p$ & 3 & $L_{q}$ & $0.0099 \mathrm{H}$ \\
\hline
\end{tabular}

\section{REFERENCES}

[1] Z. Makni and W. Zine, "Rotor position estimator based on machine learning," in IECON 2016 - 42nd Annual Conference of the IEEE Industrial Electronics Society, Oct 2016, pp. 6687-6692.

[2] D. Liang, J. Li, and R. Qu, "Sensorless control of permanent magnet synchronous machine based on second-order sliding-mode observer with online resistance estimation," IEEE Transactions on Industry Applications, vol. 53, no. 4, pp. 3672-3682, July 2017.

[3] D. Bao, Y. Wang, X. Pan, X. Wang, and K. Li, "Improved sensorless control method combining smo and mras for surface pmsm drives," in 2017 IEEE Industry Applications Society Annual Meeting, 2017.

[4] A. Yousefi-Talouki, P. Pescetto, G. Pellegrino, and I. Boldea, "Combined active flux and high-frequency injection methods for sensorless directflux vector control of synchronous reluctance machines," IEEE Transactions on Power Electronics, vol. 33, no. 3, pp. 2447-2457, March 2018.

[5] S. Medjmadj, D. Diallo, C. Delpha, and G. Yao, "A salient-pole pmsm position and speed estimation at standstill and low speed by a simplified hf injection method," in IECON 2017 - 43rd Annual Conference of the IEEE Industrial Electronics Society, Oct 2017, pp. 8317-8322.

[6] A. Matsumoto, M. Hasegawa, and S. Doki, "A novel ipmsm model for robust position sensorless control to magnetic saturation," in 2014 International Power Electronics Conference (IPEC-Hiroshima 2014 ECCE ASIA), May 2014, pp. 2445-2450.

[7] Y. D. Yoon, S. K. Sul, S. Morimoto, and K. Ide, "High-bandwidth sensorless algorithm for ac machines based on square-wave-type voltage injection," IEEE Transactions on Industry Applications, vol. 47, no. 3, pp. 1361-1370, May 2011.

[8] S. Kim, J. I. Ha, and S. K. Sul, "Pwm switching frequency signal injection sensorless method in ipmsm," IEEE Transactions on Industry Applications, vol. 48, no. 5, pp. 1576-1587, Sept 2012.

[9] T. H. Liu, S. K. Tseng, T. W. Lin, and J. L. Chen, "Sensorless ipmsm position control system using a high frequency injection method," in 2016 IEEE 2nd Annual Southern Power Electronics Conference (SPEC), Dec 2016, pp. 1-6.

[10] S. C. Yang and Y. L. Hsu, "Full speed region sensorless drive of permanent-magnet machine combining saliency-based and back-emfbased drive," IEEE Transactions on Industrial Electronics, vol. 64, no. 2, pp. 1092-1101, Feb 2017

[11] P. L. Jansen and R. D. Lorenz, "Transducerless position and velocity estimation in induction and salient ac machines," IEEE Transactions on Industry Applications, vol. 31, no. 2, pp. 240-247, Mar 1995.

[12] M. J. Corley and R. D. Lorenz, "Rotor position and velocity estimation for a salient-pole permanent magnet synchronous machine at standstill and high speeds," IEEE Transactions on Industry Applications, vol. 34, no. 4, pp. 784-789, Jul 1998.

[13] M. Schroedl, "Sensorless control of ac machines at low speed and standstill based on the ldquo;inform rdquo; method," in Industry Applications Conference, 1996. Thirty-First IAS Annual Meeting, IAS '96., Conference Record of the 1996 IEEE, vol. 1, Oct 1996, pp. 270-277 vol.1.

[14] S.-K. Sul, Control of electric machine drive systems. John Wiley \& Sons, 2011, vol. 88 .

[15] H. Li, X. Zhang, S. Yang, F. Li, and M. Ma, "Improved initial rotor position estimation of ipmsm using amplitude demodulation method based on hf carrier signal injection," in IECON 2017 - 43rd Annual Conference of the IEEE Industrial Electronics Society, Oct 2017, pp. 1996-2001.

[16] O. Wallmark, L. Harnefors, and O. Carlson, "An improved speed and position estimator for salient permanent-magnet synchronous motors," IEEE Transactions on Industrial Electronics, vol. 52, no. 1, pp. 255-262, Feb 2005.

[17] R. D. Lorenz and K. V. Patten, "High resolution velocity estimation for all digital, ac servo drives," in Conference Record of the 1988 IEEE Industry Applications Society Annual Meeting, Oct 1988, pp. 363-368 vol.1.

[18] P. Wang, Y. W. Wong, Z. Man, Z. Cao, and J. Zheng, "Sign propagation: The art behind the methodology of sliding observers," in 2017 11th Asian Control Conference (ASCC), Dec 2017, pp. 78-83.

[19] S. Shinnaka, "A new speed-varying ellipse voltage injection method for sensorless drive of permanent-magnet synchronous motors with pole saliency," IEEE Transactions on Industry Applications, vol. 44, no. 3, pp. 777-788, May 2008. 